\title{
Soliton solutions in an effective action for SU(2) Yang-Mills theory: including effects of higher-derivative term
}

\author{
N.Sawadoษ N.Shiiki \\ Department of Physics, Faculty of Science and Technology, \\ Tokyo University of Science, Noda, Chiba 278-8510, Japan
}

(Dated: June 24, 2021)

\begin{abstract}
The Skyrme-Faddeev-Niemi (SFN) model which is an $\mathrm{O}(3) \sigma$ model in three dimensional space upto fourth-order in the first derivative is regarded as a low-energy effective theory of SU(2) YangMills theory. One can show from the Wilsonian renormalization group argument that the effective action of Yang-Mills theory recovers the SFN in the infrared region. However, the thoery contains an additional fourth-order term which destabilizes the soliton solution. In this paper, we derive the second derivative term perturbatively and show that the SFN model with the second derivative term possesses soliton solutions.

PACS numbers: 11.10.Lm, 11.27.+d, 12.38.Aw, 12.38.Lg, 12.39.Dc
\end{abstract}

\section{INTRODUCTION}

The Skyrme-Faddeev-Niemi (SFN) model which is an $\mathrm{O}(3) \sigma$ model in three dimensional space upto fourthorder in the first derivative has topological soliton solutions with torus or knot-like structure. The model was initiated in 70's [1] and its interest has been extensively growing. The numerical simulations were performed in Refs. 2, 3, 4, 5, 6], the integrability was shown in Ref. 7|, and the application to the condensed matter physics [8] and the Weinberg-Salam model [9] were also considered. The recent research especially focuses on the consistency between the SFN and fundamental theories such as QCD [10, 11, 12, 13]. In those references, it is claimed that the SFN action should be deduced from the SU(2) Yang-Mills (YM) action at low energies. One can also show from the Wilsonian renormalization group argument that the effective action of Yang-Mills theory recovers the SFN in the infrared region 14|. However, the derivative expansion for slowly varying fields $\boldsymbol{n}$ upto quartic order brings an additional fourth-order term in the SFN model to destabilize the soliton solution.

Similar situations can be seen also in various topological soliton models. In the Skyrme model, the chirally invariant lagrangian with quarks produces fourth order terms after the derivative expansion and they destabilize the soliton solution [15, 16]. To recover the stability of the skyrmion, the author of Ref. [17] introduced a large number of higher order terms in the first derivative whose coefficients were determined from the coefficients of the Skyrme model by using the recursion relations. Alternatively, in Ref. 14] Gies pointed out the possibility that the second derivative order term can work as a stabilizer for the soliton.

In this paper, we examine the Gies's supposition by numerical analysis. In section [II we give an introduction

\footnotetext{
*Electronic address: sawado@ph.noda.tus.ac.jp
}

${ }^{\dagger}$ Electronic address: norikoshiiki@mail.goo.ne.jp to the Skyrme-Faddeev-Niemi model with its topological property. In section III we show how to derive the SFN model action from the SU(2) Yang-Mills theory. In section IV] soliton solutions of this truncated YM action are studied. In order to find stable soliton solutions, we introduce a second derivative term which can be derived in a perturbative manner. The naive extremization scheme, however, produce the fourth order differential equation and the model has no stable soliton solution. Failure of finding the soliton is caused by the basic feature of the second derivative field theory. In section $\mathrm{V}$ the higher derivative theory and Ostrogradski's formulation are reviewed. We show the absence of bound state in the second derivative theory using an example in quantum mechanics and introduce the perturbative treatment for the second derivative theory. In section [V] we present our numerical results. In section VII are concluding remarks.

\section{SKYRME-FADDEEV-NIEMI MODEL}

The Faddeev-Niemi conjecture for the low-energy model of SU(2) Yang-Mills theory is expressed by following effective action:

$$
S_{\mathrm{SFN}}=\Lambda \int d^{4} x\left[\frac{1}{2}\left(\partial_{\mu} \boldsymbol{n}\right)^{2}+\frac{g_{1}}{8}\left(\boldsymbol{n} \cdot \partial_{\mu} \boldsymbol{n} \times \partial_{\nu} \boldsymbol{n}\right)^{2}\right]
$$

where $\boldsymbol{n}(\boldsymbol{x})$ is a three component vector field normalized as $\boldsymbol{n} \cdot \boldsymbol{n}=1$. The mass scale $\Lambda$ is a free parameter and in this paper we set $\Lambda=1$. Stable soliton solutions exist when $g_{1}>0$.

The static field $\boldsymbol{n}(\boldsymbol{x})$ maps $\boldsymbol{n}: R^{3} \mapsto S^{2}$ and the configurations are classified by the topological maps characterized by a topological invariant $H$ called Hopf charge

$$
H=\frac{1}{32 \pi^{2}} \int A \wedge F, \quad F=d A
$$

where $F$ is the field strength and can be written as $F=$ $(\boldsymbol{n} \cdot d \boldsymbol{n} \wedge d \boldsymbol{n})$. 
The static energy $E_{\text {stt }}$ from the action (11) has a topological lower bound [18],

$$
E_{\text {stt }} \geq K H^{3 / 4}
$$

where $K=16 \pi^{2} \sqrt{g_{1}}$.

Performing numerical simulation, one can find that the static configurations for $H=1,2$ have axial symmetry 4|. Thus "the toroidal ansatz" which was studied in Ref. [3] is suitable to be imposed on these configurations. The ansatz is given by

$$
\begin{aligned}
& n_{1}=\sqrt{1-w^{2}(\eta, \beta)} \cos (N \alpha+v(\eta, \beta)), \\
& n_{2}=\sqrt{1-w^{2}(\eta, \beta)} \sin (N \alpha+v(\eta, \beta), \\
& n_{3}=w(\eta, \beta)
\end{aligned}
$$

where $(\eta, \beta, \alpha)$ is toroidal coordinates which are related to the $R^{3}$ as follows:

$$
x=\frac{a \sinh \eta \cos \alpha}{\tau}, y=\frac{a \sinh \eta \sin \alpha}{\tau}, z=\frac{a \sin \beta}{\tau}
$$

with $\tau=\cosh \eta-\cos \beta$.

The function $w(\eta, \beta)$ is subject to the boundary conditions $w(0, \beta)=1, w(\infty, \beta)=-1$ and is periodic in $\beta$. $v(\eta, \beta)$ is set to be $v(\eta, \beta)=M \beta+v_{0}(\eta, \beta)$ and $v_{0}(, \beta)$ is considered as a constant map. Equation (2) then gives $H=N M$.

In this paper we adopt a simpler ansatz than (4), which is defined by

$$
\begin{aligned}
& n_{1}=\sqrt{1-w^{2}(\eta)} \cos (N \alpha+M \beta), \\
& n_{2}=\sqrt{1-w^{2}(\eta)} \sin (N \alpha+M \beta), \\
& n_{3}=w(\eta),
\end{aligned}
$$

where $w(\eta)$ satisfies the boundary conditions $w(0)=$ $1, w(\infty)=-1$. We numerically study soliton solutions for both ansatz (4) and (6). By comparing those results, we found that this simple ansatz produces at most $10 \%$ errors and it does not affect to the property of the soliton solution.

By using (6), the static energy is written in terms of the function $w(\eta)$ as

$$
\begin{array}{r}
E_{\mathrm{stt}}=2 \pi^{2} a \int d \eta\left[\frac{\left(w^{\prime}\right)^{2}}{1-w^{2}}+\left(1-w^{2}\right) U_{M, N}(\eta)\right. \\
\left.+\frac{g_{1}}{4 a^{2}} \sinh \eta \cosh \eta\left(w^{\prime}\right)^{2} U_{M, N}(\eta)\right] \\
w^{\prime} \equiv \frac{d w}{d \eta}, \quad U_{M, N}(\eta) \equiv\left(M^{2}+\frac{N^{2}}{\sinh ^{2} \eta}\right)
\end{array}
$$

The Euler-lagrange equation of motion is then derived as

$$
\begin{aligned}
& \frac{w^{\prime \prime}}{1-w^{2}}+\frac{w w^{\prime 2}}{\left(1-w^{2}\right)^{2}}+U_{M, N}(\eta) w \\
& +\frac{g_{1}}{2 a^{2}}\left(-2 N^{2} \operatorname{coth}^{2} \eta w^{\prime}+\left(\cosh ^{2} \eta+\sinh ^{2} \eta\right) U_{M, N}(\eta) w^{\prime}\right. \\
& \left.+\sinh \eta \cosh \eta U_{M, N}(\eta) w^{\prime \prime}\right)=0 .
\end{aligned}
$$

The variation with respect to $a$ produces the equation for variable $a$. Soliton solutions are obtained by solving the equations for $a$ as well as for $w$.

\section{EFFECTIVE ACTION IN THE YANG-MILLS THEORY WITH CFNS DECOMPOSITION}

In this section, we briefly review how to derive the SFN effective action from the action of SU(2) Yang-Mills theory in the infrared limit 12, 14]. For the gauge fields $\boldsymbol{A}_{\mu}$, the Cho-Faddeev-Niemi-Shabanov decomposition is applied [10, 11, 12, 13]

$$
\boldsymbol{A}_{\mu}=\boldsymbol{n} C_{\mu}+\left(\partial_{\mu} \boldsymbol{n}\right) \times \boldsymbol{n}+\boldsymbol{W}_{\mu} .
$$

The first two terms are the "electric" and "magnetic" Abelian connection, and $\boldsymbol{W}_{\mu}$ are chosen so as to orthogonal to $\boldsymbol{n}, \boldsymbol{W}_{\mu} \cdot \boldsymbol{n}=0$. Obviously, the degrees of freedom on the left- and right-hand side of Eq.(8) do not match. While the LHS describes $3_{\text {color }} \times 4_{\text {Lorentz }}=12$, the RHS is comprised of $\left(C_{\mu}:\right) 4_{\text {Lorentz }}+(\boldsymbol{n}:) 2_{\text {color }}+\left(\boldsymbol{W}_{\mu}\right.$ : ) $3_{\text {color }} \times 4_{\text {Lorentz }}-4_{\boldsymbol{n}} \cdot \boldsymbol{W}_{\mu}=0=14$ degrees freedom. Shabanov introduced in his paper [12] the following constraint

$$
\chi\left(\boldsymbol{n}, C_{\mu}, \boldsymbol{W}_{\mu}\right)=0, \text { with } \boldsymbol{\chi} \cdot \boldsymbol{n}=0 .
$$

The generating functional of YM theory can be written by using Eq. (8) as

$$
\mathcal{Z}=\int \mathcal{D} \boldsymbol{n} \mathcal{D} C \mathcal{D} \boldsymbol{W} \delta(\boldsymbol{\chi}) \Delta_{\mathrm{FP}} \Delta_{\mathrm{S}} e^{-S_{\mathrm{YM}}-S_{\mathrm{gf}}}
$$

$\Delta_{\mathrm{FP}}$ and $S_{\mathrm{gf}}$ are the Faddeev-Popov determinant and the gauge fixing action respectively, and Shabanov introduced another determinant $\Delta_{\mathrm{S}}$ corresponding to the condition $\chi=0$. YM and the gauge fixing action is given by

$$
S_{\mathrm{YM}}+S_{\mathrm{gf}}=\int d^{4} x\left[\frac{1}{4 g^{2}} \boldsymbol{F}_{\mu \nu} \cdot \boldsymbol{F}_{\mu \nu}+\frac{1}{2 \alpha_{\mathrm{g}} g^{2}}\left(\partial_{\mu} \boldsymbol{A}_{\mu}\right)^{2}\right] .
$$

Inserting Eq. (8) into the action, one obtains the vacuum functional

$$
\begin{aligned}
\mathcal{Z} & =\int \mathcal{D} \boldsymbol{n} e^{-\mathcal{S}_{\mathrm{eff}}(\boldsymbol{n})} \\
& =\int \mathcal{D} \boldsymbol{n} e^{-\mathcal{S}_{\mathrm{cl}}(\boldsymbol{n})} \int \mathcal{D} \tilde{C} \mathcal{D} \boldsymbol{W}_{\mu} \Delta_{\mathrm{FP}} \Delta_{\mathrm{S}} \delta(\chi) \\
& \times e^{-\left(1 / 2 g^{2}\right) \int\left(\tilde{C}_{\mu} M_{\mu \nu}^{C} \tilde{C}_{\nu}+\boldsymbol{W}_{\mu} \bar{M}_{\mu \nu}^{W} \boldsymbol{W}_{\nu}+2 C_{\nu} K_{\nu}^{C}+2 \boldsymbol{W}_{\mu} \cdot \boldsymbol{K}_{\mu}^{W}\right)}
\end{aligned}
$$

with

$$
\begin{aligned}
& M_{\mu \nu}^{C}=-\partial^{2} \delta_{\mu \nu}+\partial_{\mu} \boldsymbol{n} \cdot \partial_{\nu} \boldsymbol{n}, \\
& M_{\mu \nu}^{\boldsymbol{W}}=-\partial^{2} \delta_{\mu \nu}-\partial_{\mu} \boldsymbol{n} \otimes \partial_{\nu} \boldsymbol{n}+\partial_{\nu} \boldsymbol{n} \otimes \partial_{\mu} \boldsymbol{n}, \\
& \boldsymbol{Q}_{\mu \nu}^{C}=\partial_{\mu} \boldsymbol{n} \partial_{\nu}+\partial_{\nu} \boldsymbol{n} \partial_{\mu}+\partial_{\mu} \partial_{\nu} \boldsymbol{n}, \\
& K_{\mu \nu}^{C}=\partial_{\nu}\left(\boldsymbol{n} \cdot \partial_{\nu} \boldsymbol{n} \times \partial_{\mu} \boldsymbol{n}\right)+\partial_{\mu} \boldsymbol{n} \cdot \partial^{2} \boldsymbol{n} \times \boldsymbol{n}, \\
& \boldsymbol{K}_{\mu \nu}^{\boldsymbol{W}}=\partial_{\mu}\left(\boldsymbol{n} \times \partial^{2} \boldsymbol{n}\right), \quad\left(\text { in gauge } \alpha_{\mathrm{g}}=1\right)
\end{aligned}
$$


and

$$
\begin{aligned}
& \bar{M}_{\mu \nu}^{\boldsymbol{W}}:=M_{\mu \nu}^{\boldsymbol{W}}-\boldsymbol{Q}_{\mu s} M_{s \lambda}^{C_{s \lambda}^{-1}} \boldsymbol{Q}_{\lambda \nu}, \\
& \tilde{C}_{\mu}=C_{\mu}+\boldsymbol{W}_{s} \cdot \boldsymbol{Q}_{s \lambda} M_{\lambda \mu}^{C_{\lambda \mu}^{-1}} .
\end{aligned}
$$

The classical action of $\boldsymbol{n}$ including the gauge fixing term is given by

$$
\mathcal{S}_{\mathrm{cl}}=\int d^{4} x\left[\frac{1}{4 g^{2}}\left(\partial_{\mu} \boldsymbol{n} \times \partial_{\nu} \boldsymbol{n}\right)^{2}+\frac{1}{2 \alpha_{\mathrm{g}} g^{2}}\left(\partial^{2} \boldsymbol{n} \times \boldsymbol{n}\right)^{2}\right] .
$$

The $\delta$ functional is expressed by its Fourier transform

$$
\delta(\boldsymbol{\chi})=\int \mathcal{D} \boldsymbol{\phi} e^{-i \int\left(\boldsymbol{\phi} \cdot \partial \boldsymbol{W}_{\mu}+\boldsymbol{\phi} \cdot C_{\mu} \boldsymbol{n} \times \boldsymbol{W}_{\mu}+(\boldsymbol{\phi} \cdot \boldsymbol{n})\left(\partial_{\mu} \boldsymbol{n} \cdot \boldsymbol{W}_{\mu}\right)\right)} .
$$

Integrating over $C, \boldsymbol{W}, \boldsymbol{\phi}$, we finally obtain

$$
\begin{aligned}
e^{-S_{\text {eff }}} & =e^{-S_{\mathrm{cl}}} \Delta_{\mathrm{FP}} \Delta_{\mathrm{S}}\left(\operatorname{det} M^{C}\right)^{-1 / 2}\left(\operatorname{det} \bar{M}^{\boldsymbol{W}}\right)^{-1 / 2} \\
& \times\left(\operatorname{det}-Q_{\mu}^{\phi}\left(\bar{M}^{\boldsymbol{W}}\right)_{\mu \nu}^{-1} Q_{\nu}^{\phi}\right)^{-1 / 2}
\end{aligned}
$$

where several nonlocal terms and the higher derivative components have been neglected.

We perform the derivative expansion for the four determinants in Eq. (16) under the following assumptions

(i) the theory is valid for the momenta $p$ with $k<p<$ $\Lambda(k, \Lambda$ are infrared and ultraviolet cut-off $)$

(ii) $|\partial \boldsymbol{n}| \ll k$

(iii) the higher derivative terms, such as $\partial^{2} \boldsymbol{n}$ are omitted.

The effective action is then given by

$$
\begin{aligned}
S_{\mathrm{eff}}=\int d^{4} x & {\left[\frac{1}{2}\left(\partial_{\mu} \boldsymbol{n}\right)^{2}+\frac{g_{1}}{8}\left(\partial_{\mu} \boldsymbol{n} \times \partial_{\nu} \boldsymbol{n}\right)^{2}\right.} \\
+ & \left.\frac{g_{2}}{8}\left(\partial_{\mu} \boldsymbol{n}\right)^{4}\right] .
\end{aligned}
$$

For $g_{1}>0$ and $g_{2}=0$, the action is identical to the FSN effective action (11).

In order to get the stable soliton solutions, $g_{2}$ must be positive [3]. However, $g_{2}$ is found to be negative according to the above analysis. Therefore we consider higherderivative terms and investigate if the model with the higher-derivatives possess soliton solutions.

\section{SEARCH FOR THE STABLE SOLITON SOLUTIONS (1)}

The static energy is derived from Eq.(17) as

$$
\begin{aligned}
E_{\mathrm{stt}} & =\int d^{3} x\left[\frac{1}{2}\left(\partial_{i} \boldsymbol{n}\right)^{2}+\frac{g_{1}}{8}\left(\partial_{i} \boldsymbol{n} \times \partial_{j} \boldsymbol{n}\right)^{2}+\frac{g_{2}}{8}\left(\partial_{i} \boldsymbol{n}\right)^{4}\right] \\
& :=E_{2}(\boldsymbol{n})+E_{4}^{(1)}(\boldsymbol{n})+E_{4}^{(2)}(\boldsymbol{n}) .
\end{aligned}
$$

A spatial scaling behaviour of the static energy, so called Derrick's scaling argument, can be applied to examine the stability of the soliton 19]. Considering the map $\boldsymbol{x} \mapsto \boldsymbol{x}^{\prime}=\mu \boldsymbol{x}(\mu>0)$, with $\boldsymbol{n}^{(\mu)} \equiv \boldsymbol{n}(\mu \boldsymbol{x})$, the static energy scales as

$$
\begin{aligned}
e(\mu) & =E_{\mathrm{stt}}\left(\boldsymbol{n}^{(\mu)}\right) \\
& =E_{2}\left(\boldsymbol{n}^{(\mu)}\right)+E_{4}^{(1)}\left(\boldsymbol{n}^{(\mu)}\right)+E_{4}^{(2)}\left(\boldsymbol{n}^{(\mu)}\right) \\
& =\frac{1}{\mu} E_{2}(\boldsymbol{n})+\mu\left(E_{4}^{(1)}(\boldsymbol{n})+E_{4}^{(2)}(\boldsymbol{n})\right) .
\end{aligned}
$$

Derrick's theorem states that if the function $e(\mu)$ has no stationary point, the theory has no static solutions of the field equation with finite density, other than the vacuum. Conversely, if $e(\mu)$ has stationary point, the possibility of having finite energy soliton solutions is not excluded. Eq. (19) is stationary at $\mu=\sqrt{E_{2} /\left(E_{4}^{(1)}+E_{4}^{(2)}\right)}$. Then, the following inequality

$$
\begin{aligned}
& g_{1}\left(\partial_{i} \boldsymbol{n} \times \partial_{j} \boldsymbol{n}\right)^{2}+g_{2}\left(\partial_{i} \boldsymbol{n}\right)^{2}\left(\partial_{j} \boldsymbol{n}\right)^{2} \\
& =g_{1}\left(\partial_{i} \boldsymbol{n}\right)^{2}\left(\partial_{j} \boldsymbol{n}\right)^{2}-g_{1}\left(\partial_{i} \boldsymbol{n} \cdot \partial_{j} \boldsymbol{n}\right)^{2}+g_{2}\left(\partial_{i} \boldsymbol{n}\right)^{2}\left(\partial_{j} \boldsymbol{n}\right)^{2} \\
& \geqq g_{2}\left(\partial_{i} \boldsymbol{n} \cdot \partial_{j} \boldsymbol{n}\right)^{2} \quad\left(\because\left(\partial_{i} \boldsymbol{n}\right)^{2}\left(\partial_{j} \boldsymbol{n}\right)^{2} \geqq\left(\partial_{i} \boldsymbol{n} \cdot \partial_{j} \boldsymbol{n}\right)^{2}\right)
\end{aligned}
$$

ensures the possibility of existence of the stable soliton solutions for $g_{2} \geqq 0$. As mentioned in the section 【II $g_{2}$ should be negative at least within our derivative expansion analysis of YM theory.

A promising idea to tackle the problem was suggested by Gies 14]. He considered the following type of effective action, accompanying second derivative term

$$
\begin{aligned}
S_{\mathrm{eff}}=\int d^{4} x & {\left[\frac{1}{2}\left(\partial_{\mu} \boldsymbol{n}\right)^{2}+\frac{g_{1}}{8}\left(\partial_{\mu} \boldsymbol{n} \times \partial_{\nu} \boldsymbol{n}\right)^{2}\right.} \\
& \left.-\frac{g_{2}}{8}\left(\partial_{\mu} \boldsymbol{n}\right)^{4}+\frac{g_{2}}{8}\left(\partial^{2} \boldsymbol{n} \cdot \partial^{2} \boldsymbol{n}\right)\right] .
\end{aligned}
$$

Here we choose positive value of $g_{2}$ and assign the explicit negative sign to the third term. In principle, it is possible to estimate the second derivative term by the derivative expansion without neglecting throughout the calculation. The calculation is, however, very laborious and hence we show only one simple example of the $C$ determinant. The determinant is real and thus it is expanded as follows

$$
\begin{aligned}
& \log \left(\operatorname{det} M^{C}\right)^{-1 / 2}=-\frac{1}{2} \operatorname{Tr} \log \left(-\partial^{2}+\partial_{\mu} \boldsymbol{n} \cdot \partial_{\mu} \boldsymbol{n}\right) \\
& \rightarrow-\frac{1}{4} \operatorname{Tr} \log \left[\partial^{4}-2(\partial \boldsymbol{n})^{2} \partial^{2}+(\partial \boldsymbol{n})^{4}-\partial^{2}(\partial \boldsymbol{n})^{2}\right] \\
&=-\frac{1}{4} \operatorname{Tr} \log \left(\partial^{4}\right) \\
&-\frac{1}{4} \operatorname{Tr} \log \left[1-2 \frac{\left(\partial \boldsymbol{n}^{2}\right)}{\partial^{2}}+\frac{(\partial \boldsymbol{n})^{4}}{\partial^{4}}-\frac{\partial^{2}(\partial \boldsymbol{n})^{2}}{\partial^{4}}\right] \\
&=-\frac{1}{4} \operatorname{Tr} \log \left(\partial^{4}\right) \\
&-\frac{1}{4} \operatorname{Tr}\left[-2 \frac{\left(\partial \boldsymbol{n}^{2}\right)}{\partial^{2}}+\frac{(\partial \boldsymbol{n})^{4}}{\partial^{4}}-\frac{\partial^{2}(\partial \boldsymbol{n})^{2}}{\partial^{4}}\right] \\
&+\frac{1}{8} \operatorname{Tr}\left[-2 \frac{(\partial \boldsymbol{n})^{2}}{\partial^{2}}\right]^{2}+O\left((\partial \boldsymbol{n})^{6}\right)
\end{aligned}
$$


where we have defined $\partial_{\mu} \boldsymbol{n} \cdot \partial_{\mu} \boldsymbol{n} \rightarrow(\partial \boldsymbol{n})^{2},\left(\partial_{\mu} \boldsymbol{n} \cdot \partial_{\nu} \boldsymbol{n}\right)^{2} \rightarrow$ $(\partial \boldsymbol{n})^{4}$. Employing the integral formulas [14]

$$
\begin{aligned}
& \int_{[k, \Lambda]} \frac{d^{4} p}{(2 \pi)^{4}} \frac{1}{p^{2}}=\frac{1}{16 \pi^{2}}\left(\Lambda^{2}-k^{2}\right), \\
& \int_{[k, \Lambda]} \frac{d^{4} p}{(2 \pi)^{4}} \frac{1}{p^{4}}=\frac{1}{8 \pi^{2}} \log \frac{\Lambda}{k}
\end{aligned}
$$

together with the equality (up to second derivative)

$$
\partial^{2}\left(\partial_{\mu} \boldsymbol{n} \cdot \partial_{\mu} \boldsymbol{n}\right)=-\partial^{2}\left(\boldsymbol{n} \cdot \partial^{2} \boldsymbol{n}\right)=-\partial^{2} \boldsymbol{n} \cdot \partial^{2} \boldsymbol{n},
$$

we obtain the form for the $C$ determinant

$$
\begin{aligned}
& \log \left(\operatorname{det} M^{C}\right)^{-1 / 2}= \\
& -\frac{1}{32 \pi^{2}} \int d^{4} x\left[\left(\Lambda^{2}-k^{2}\right)\left(\partial_{\mu} \boldsymbol{n}\right)^{2}-\log \frac{\Lambda}{k}\left(\partial_{\mu} \boldsymbol{n} \times \partial_{\nu} \boldsymbol{n}\right)^{2}\right. \\
& \left.\quad+\log \frac{\Lambda}{k}\left(\partial_{\mu} \boldsymbol{n}\right)^{4}-\log \frac{\Lambda}{k}\left(\partial^{2} \boldsymbol{n} \cdot \partial^{2} \boldsymbol{n}\right)\right] .
\end{aligned}
$$

The other determinants can be estimated in a similar manner.

The static energy of Eq. (21) with the ansatz (6) is written as

$$
\begin{aligned}
& E_{\mathrm{stt}}= 2 \pi^{2} a \int d \eta\left[\frac{\left(w^{\prime}\right)^{2}}{1-w^{2}}+\left(1-w^{2}\right) U_{M, N}(\eta)\right. \\
&+\frac{g_{1}}{4 a^{2}} \sinh \eta \cosh \eta\left(w^{\prime}\right)^{2} U_{M, N}(\eta)-\frac{g_{2}}{4 a^{2}} \sinh \eta \cosh \eta\left[\frac{\left(w^{\prime}\right)^{2}}{1-w^{2}}+\left(1-w^{2}\right) U_{M, N}(\eta)\right]^{2} \\
&+\frac{g_{2}}{4 a^{2}}\left[\left(\operatorname{coth} \eta+\sinh ^{2} \eta-\sinh \eta \cosh \eta\right) \frac{\left(w^{\prime}\right)^{2}}{1-w^{2}}\right. \\
&+\left(\sinh \eta \cosh \eta-\sinh ^{2} \eta\right)\left(1-w^{2}\right) M^{2} \\
&+2\left\{\frac{w\left(w^{\prime}\right)^{3}}{\left(1-w^{2}\right)^{2}}+\frac{w^{\prime} w^{\prime \prime}}{1-w^{2}}+w^{\prime} U_{M, N}(\eta)\right\} \\
&+\sinh \eta \cosh \eta\left\{\frac { 1 } { 1 - w ^ { 2 } } \left[\frac{\left(w^{\prime}\right)^{2}}{1-w^{2}}+w w^{\prime \prime}\right.\right. \\
&\left.\left.\left.+\left(1-w^{2}\right) U_{M, N}(\eta)\right]^{2}+\left(w^{\prime \prime}\right)^{2}\right\}\right]
\end{aligned}
$$

The Euler-Lagrange equation of motion is derived by

$$
-\frac{d^{2}}{d \eta^{2}}\left(\frac{\partial E_{\mathrm{stt}}}{\partial w^{\prime \prime}}\right)+\frac{d}{d \eta}\left(\frac{\partial E_{\mathrm{stt}}}{\partial w^{\prime}}\right)-\frac{\partial E_{\mathrm{stt}}}{\partial w}=0
$$

which is too complicated and thus we adopt the following notation

$$
\begin{aligned}
f_{0}\left(w, w^{\prime}, w^{\prime \prime}\right)+g_{1} f_{1}\left(w, w^{\prime}, w^{\prime \prime}\right) & \\
& +g_{2} f_{2}\left(w, w^{\prime}, w^{\prime \prime}, w^{(3)}, w^{(4)}\right)=0 .
\end{aligned}
$$

Here $w^{(3)}, w^{(4)}$ represent the third and the fourth derivative with respect to $\eta$. The first two terms of Eq.(26) are identical to those in Eq.(7).
Unfortunately, we could not find out stable soliton solutions from Eq. (26) for any value of $g_{2}$.

From the relation

$$
\int d^{4} x\left[\left(\partial^{2} \boldsymbol{n} \cdot \partial^{2} \boldsymbol{n}\right)-\left(\partial_{\mu} \boldsymbol{n}\right)^{4}\right]=\int d^{4} x\left(\partial^{2} \boldsymbol{n} \times \boldsymbol{n}\right)^{2},
$$

one easily finds that the static energy obtained from the last two terms in Eq. (21)

$$
\tilde{E}_{4}^{(2)}=\int d^{3} x\left(\partial^{2} \boldsymbol{n} \times \boldsymbol{n}\right)^{2}
$$

gives the positive contribution. The total static energy is stationary at $\mu=\sqrt{E_{2} /\left(E_{4}^{(1)}+\tilde{E}_{4}^{(2)}\right)}$ and hence the possibility of existence of soliton solutions is not excluded. And also, the positivity of Eq.(28) does not spoil the lower bound (3) of original SFN and the possibility is still not excluded, too.

Therefore, we suspect that the absence of the stable soliton is caused by the fact that higher derivative theory has no lower bound state. We shall investigate the lower bound in the higher derivative theory in detail in the next section.

\section{HIGHER DERIVATIVE THEORY}

In this section, we address the basic problems in the higher derivative theory [21, 22, 23, 24, 25] which essentially falls into two categories. The first problem concerns the increase in the number of degrees of freedom. For example, if the theory contains second derivative terms, the equation of motion becomes up to the order in the fourth derivative. Thus, four parameters are required for the initial conditions. If one considers more higher order terms, the situation gets worse. However, this is not serious problem for our study because our concern is the existence of static soliton solutions. The second problem is that the actions of the theory are not bounded from below. This feature makes the higher derivative theories unstable.

The lagrangian and the hamiltonian formalism with higher derivative was firstly developed by Ostrogradski [20]. We consider the lagrangian containing up to $n$th order derivatives

$$
S=\int d t \mathcal{L}\left(q, \dot{q}, \cdots, q^{(n)}\right)
$$

Taking the variation of the action $\delta S=0$ leads the Eulerlagrange equation of motion

$$
\sum_{i=0}^{n}(-1)^{i} \frac{d^{i}}{d t^{i}}\left(\frac{\partial \mathcal{L}}{\partial q^{(i)}}\right)=0 .
$$

The hamiltonian is obtained by introducing $n$ generalized momenta

$$
p_{i}=\sum_{j=i+1}^{n}(-1)^{j-i-1} \frac{d^{j-i-1}}{d t^{j-i-1}}\left(\frac{\partial \mathcal{L}}{\partial q^{(j)}}\right), i=1, \cdots, n,
$$


or

$$
\begin{aligned}
& p_{n}=\frac{\partial \mathcal{L}}{\partial q^{(n)}}, \\
& p_{i}=\frac{\partial \mathcal{L}}{\partial q^{(i)}}-\frac{d}{d t} p_{i+1}, \quad i=1, \cdots, n-1,
\end{aligned}
$$

and $n$ independent variables

$$
\begin{aligned}
& q_{1} \equiv q, \\
& q_{i} \equiv q^{(i-1)}, \quad(i=2, \cdots, n) .
\end{aligned}
$$

The lagrangian now depends on the $n$ coordinates $q_{i}$ and on the first derivative $\dot{q}_{n}=q^{(n)}$. The hamiltonian is defined as

$$
\mathcal{H}\left(q_{i}, p_{i}\right)=\sum_{i=1}^{n} p_{i} \dot{q}_{i}-\mathcal{L}=\sum_{i=1}^{n-1} p_{i} q_{i+1}+p_{n} \dot{q}_{n}-\mathcal{L}
$$

The canonical equations of motion turn out to be

$$
\dot{q}_{i}=\frac{\partial \mathcal{H}}{\partial p_{i}}, \quad \dot{p}_{i}=-\frac{\partial \mathcal{H}}{\partial q_{i}} .
$$

We consider a simple example including second derivative term [25], defined as

$$
\mathcal{L}=\frac{1}{2}\left(1+\varepsilon^{2} \omega^{2}\right) \dot{q}^{2}-\frac{1}{2} \omega^{2} q^{2}-\frac{1}{2} \varepsilon^{2} \ddot{q}^{2},
$$

where constant $\epsilon$ works as a coupling constant of second derivative term. The equation of motion is

$$
\left(1+\varepsilon^{2} \omega^{2}\right) \ddot{q}+\omega^{2} q+\varepsilon^{2} q^{(4)}=0 .
$$

From Eq. (32), one gets

$$
\begin{aligned}
& \pi_{\dot{q}}=\frac{\partial \mathcal{L}}{\partial \ddot{q}}=-\varepsilon^{2} \ddot{q}, \\
& \pi_{q}=\frac{\partial \mathcal{L}}{\partial \dot{q}}-\frac{d}{d t}\left(\frac{\partial \mathcal{L}}{\partial \ddot{q}}\right)=\left(1+\varepsilon^{2} \omega^{2}\right) \dot{q}+\varepsilon^{2} \dddot{q} .
\end{aligned}
$$

Thus the hamiltonian becomes

$$
\begin{aligned}
\mathcal{H} & =\dot{x} \pi_{q}+\ddot{q} \pi_{\dot{q}}-\mathcal{L} \\
& =\dot{q} \pi_{q}-\frac{1}{2 \varepsilon^{2}} \pi_{\dot{q}}^{2}-\frac{1}{2}\left(1+\varepsilon^{2} \omega^{2}\right) \dot{q}^{2}+\frac{1}{2} \omega^{2} q^{2} .
\end{aligned}
$$

We introduce the new canonical variables

$$
\begin{aligned}
& q_{+}=\frac{1}{\omega \sqrt{1-\varepsilon^{2} \omega^{2}}}\left(\varepsilon^{2} \omega^{2} \dot{q}-\pi_{q}\right), \\
& p_{+}=\frac{w}{\sqrt{1-\varepsilon^{2} \omega^{2}}}\left(q-\pi_{\dot{q}}\right), \\
& q_{-}=\frac{\varepsilon}{\sqrt{1-\varepsilon^{2} \omega^{2}}}\left(\dot{q}-\pi_{q}\right), \\
& p_{-}=\frac{1}{\varepsilon \sqrt{1-\varepsilon^{2} \omega^{2}}}\left(\varepsilon^{2} \omega^{2} q-\pi_{\dot{q}}\right),
\end{aligned}
$$

and the hamiltonian has of the form by using these variables

$$
\mathcal{H} \rightarrow \frac{1}{2}\left(p_{+}^{2}+\omega^{2} q_{+}^{2}\right)-\frac{1}{2}\left(p_{-}^{2}+\frac{1}{\varepsilon^{2}} q_{-}^{2}\right) .
$$

The corresponding energy spectra is then given by

$$
E=\left(n+\frac{1}{2}\right) \omega-\left(m+\frac{1}{2}\right) \frac{1}{\varepsilon}, \quad n, m=0,1,2, \cdots
$$

One can see that in the limit $\epsilon \rightarrow 0$ the energy goes to negative infinity rather than approaching to the harmonic oscillator energy eigenstates.

To obtain physically meaningful solutions, we employ the perturbative analysis where the solution is expanded in terms of the small coupling constant and the EulerLagrange equation of motion is replaced with the corresponding perturbative equation. The solutions of the equations of motion that are ill behaved in the limit $\epsilon \rightarrow 0$ are excluded from the very beginning [23, 24, 25].

We assume that the solution of Eq.(37) can be written as

$$
q_{\text {pert }}(t)=\sum_{n=0}^{\infty} \epsilon^{n} q(t)
$$

Substituting Eq. (41) into Eq. (37) and taking time derivatives of these equations, we obtain the constraints for higher derivative terms

$$
\begin{aligned}
& O\left(\epsilon^{0}\right) \\
& \text { equation: } \ddot{q}_{0}+\omega^{2} q_{0}=0 \text {, } \\
& \text { constraints : } \dddot{q}_{0}=-\omega^{2} \dot{q}_{0}, \dddot{q}_{0}=\omega^{4} q_{0} \text {. } \\
& O\left(\epsilon^{2}\right) \\
& \text { equation: } \ddot{q}_{2}+\omega^{2} \ddot{q}_{0}+\omega^{2} q_{2}+\dddot{\dddot{q}}_{0}=0 \text {, } \\
& \Rightarrow \ddot{q}_{2}+\omega^{2} q_{2}=0, \quad(\text { using (42), (43)) } \\
& \text { constraints : } \dddot{q}_{2}=-\omega^{2} \dot{q}_{2}, \dddot{q}_{2}=\omega^{4} q_{2} \text {. } \\
& O\left(\epsilon^{4}\right) \\
& \text { equation: } \ddot{q}_{4}+\omega^{2} \ddot{q}_{2}+\omega^{2} q_{4}+\dddot{\dddot{q}}_{2}=0 \text {, } \\
& \Rightarrow \ddot{q}_{4}+\omega^{2} q_{4}=0, \quad \text { (using (44), (45)), } \\
& \text { constraints : } \dddot{q}_{4}=-\omega^{2} \dot{q}_{4}, \dddot{q}_{4}=\omega^{4} q_{4} \text {. }
\end{aligned}
$$

Combining these results, we find the perturbative equation of motion up to $O\left(\epsilon^{4}\right)$

$$
\ddot{q}_{\text {pert }}+\omega^{2} q_{\text {pert }}=O\left(\epsilon^{6}\right) .
$$

which is the equation for harmonic oscillator.

\section{SEARCH FOR THE STABLE SOLITON SOLUTIONS (2) - PERTURBATIVE} ANALYSIS -

Let us employ the perturbative method introduced in the last section to our problem. We assume that $g_{2}$ is relatively small and can be considered as a perturbative coupling constant. Thus, the perturbative solution is written by a power series in $g_{2}$

$$
w(\eta)=\sum_{n=0}^{\infty} g_{2}^{n} w_{n}(\eta)
$$




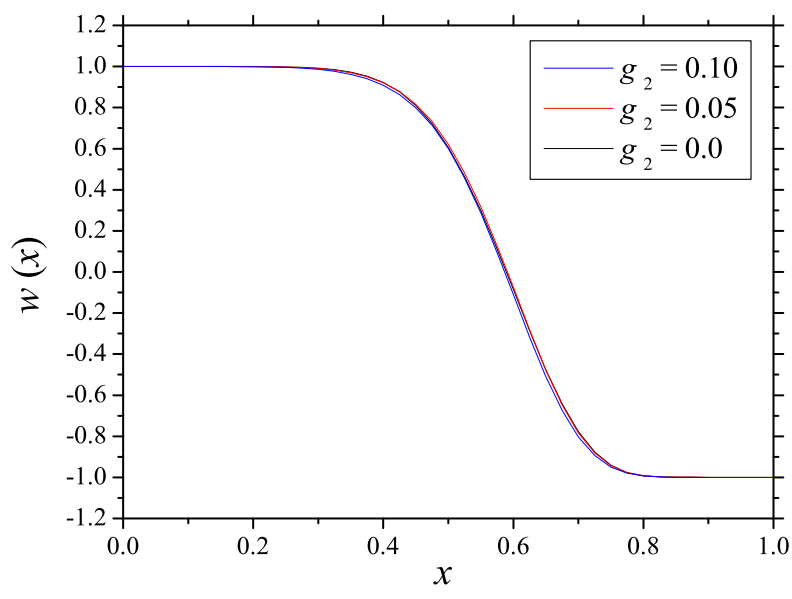

FIG. 1: The function $w(\eta)$ for $g_{1}=0.4, g_{2}=0,0.05,0.1$ (the rescaling radial coordinate $x=\eta /(1-\eta)$ is used).

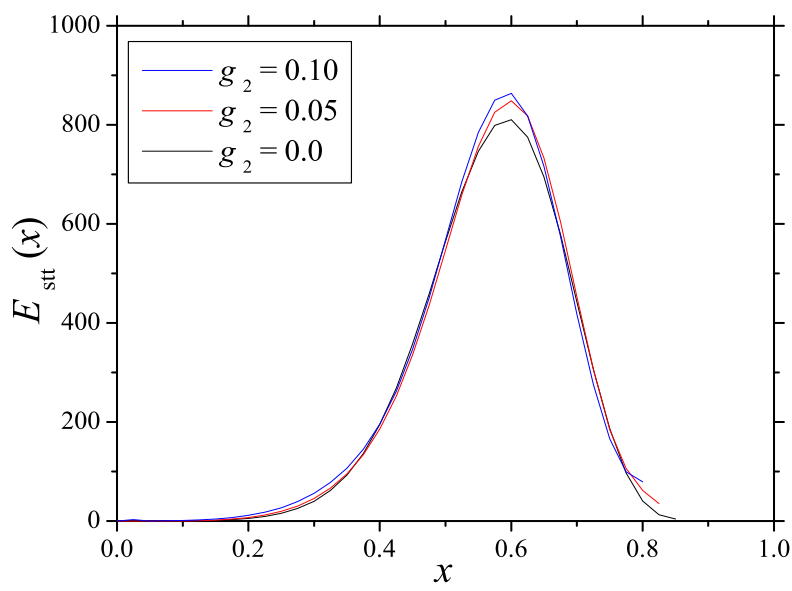

FIG. 2: The energy density for $g_{1}=0.4, g_{2}=0,0.05,0.1$.

Substituting Eq.(49) into Eq.(26), we obtain the classical field equation in $O\left(g_{2}^{0}\right)$

$$
f_{0}\left(w_{0}, w_{0}^{\prime}, w_{0}^{\prime \prime}\right)+g_{1} f_{1}\left(w_{0}, w_{0}^{\prime}, w_{0}^{\prime \prime}\right)=0 .
$$

Taking derivatives for both sides in Eq.(50) and solving for $w_{0}^{\prime \prime}, w_{0}^{(3)}, w_{0}^{(4)}$ read the following form of the constraint equations for higher derivatives

$$
w_{0}^{(i)}=F^{(i)}\left(w_{0}, w_{0}^{\prime}\right), \quad i=2,3,4 .
$$

The equation in $O\left(g_{2}^{1}\right)$ can be written as

$$
\left(f_{0}+g_{2} f_{1}\right)_{O\left(g_{2}^{1}\right)}+f_{2}\left(w_{0}, w_{0}^{\prime}, w_{0}^{\prime \prime}, w_{0}^{(3)}, w_{0}^{(4)}\right)=0 .
$$

Substituting the constraint equations (51) into Eq.(52) and eliminate the higher derivative terms, one can obtain

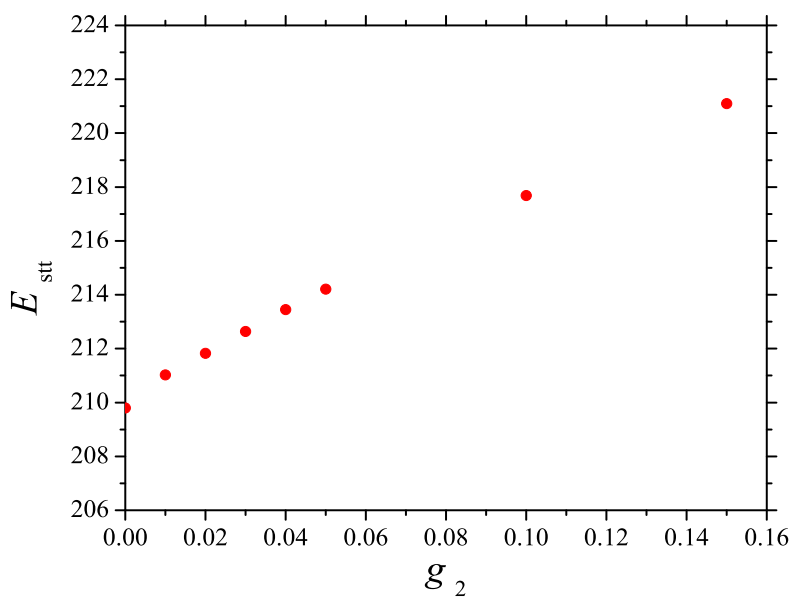

FIG. 3: The energy as a function of $g_{2}\left(g_{1}=0.4\right)$.

the perturbative equation of motion

$$
f_{0}\left(w, w^{\prime}, w^{\prime \prime}\right)+g_{1} f_{1}\left(w, w^{\prime}, w^{\prime \prime}\right)+g_{2} \tilde{f}_{2}\left(w, w^{\prime}\right)=O\left(g_{2}^{2}\right) .
$$

Now Eq. (53) has topological soliton solutions. Our results of the estimated function $w(\eta)$ and the energy density are displayed in Figs 112 (In all figures, we show the results for the case of Hopf charge $H=2 ; N=2, M=1$ ). We have small changes for varying the coupling constant $g_{2}$. The dependence of the $g_{2}$ for the total energy is shown in Fig 3 It can be seen that the change is moderate with respect to $g_{2}$.

\section{SUMMARY}

In this paper we have studied the Skyrme-FaddeevNiemi model and its extensions by introducing the reduction scheme of the $\mathrm{SU}(2)$ Yang-Mills theory to the corresponding low-energy effective model. The requirement of consistency between the low-energy effecive action of the YM and the SFN type model lead us to take into account second derivative terms in the action. However, we found that such an action including the second derivative terms does not have stable soliton solutions. This is due to the absence of the energy bound in higher derivative theory. This fact inspired us to employ the perturbative analysis to our effective action. Within the perturbative analysis, we were able to obtain the topological soliton solutions.

Our analysis is based on perturbation and the coupling constant $g_{2}$ is assumed to be small. However, Wilsonian renormalization analysis of YM theory [14] suggest that the coupling constants $g_{1}, g_{2}$ (and the mass scale parameter $\Lambda$ ) depend on the renormalization group time $t=\log k / \Lambda(k, \Lambda$ are infrared, ultraviolet cutoff parameter) and those are almost comparable. To improve the 
analysis, we could perform the next order of perturbation, but it is tedius and spoils the simplicity of the FSN model unfortunately.

It should be noted that our solutions do not much differ from the solution of original SFN model, at least in the perturbative regime. We suspect that some appropriate truncation (like "extra fourth order term + second derivative term") always supply the stable solutions that are close to the original SFN model. Thus we conclude that the topological soliton model comprised of the "ki-

[1] L.Faddeev, Report No.IAS Print-75-QS70, 1975.

[2] L.Faddeev and A.Niemi, Nature (London) 387, 58 (1997).

[3] Jens Gladikowski and Meik Hellmund, Phys. Rev.D 56, 5194 (1997).

[4] Richard A.Battye and Paul M.Sutcliffe, Proc. R. Soc. Lond. A 455, (1999.)

[5] Jarmo Hietarinta and Petri Salo, Phys. Lett. B 451,60 (1999).

[6] Jarmo Hietarinta and Petri Salo, Phys. Rev.D 62, 081701(R) (2000).

[7] H.Aratyn, L.A.Ferreira, A.H.Zimerman, Phys. Lett. B 456,162 (1999).

[8] Egor Babaev, Ludvig Faddeev and Antti J.Niemi, Phys. Rev.B 65, 100512 (2002).

[9] B.A.Fayzullaev, M.M.Musakhanov, D.G.Pak, and M.Siddikov, Phys. Lett. B 609, 442 (2005).

[10] Ludvig Faddeev and Antti J.Niemi, Phys. Rev. Lett. 22, 1624 (1999).

[11] Edwin Langmann, Antti J.Niemi, Phys. Lett. B 463, 252 (1999).

[12] Sergei V. Shabanov, Phys. Lett. B 458, 322 (1999). netic term + a special fourth order term" like SFN model is a good approximation.

\section{Acknowledgments}

The authors thank to Kei-Ichi Kondo for drawing our attention to the coefficient problem of this model.

[13] Y.M.Cho, H.W.Lee, D.G.Pak, Phys. Lett. B 525, 347 (2002).

[14] Holger Gies, Phys. Rev.D 63, 125023 (2001).

[15] Avinash Dhar, R.Shankar, and Spenta R.Wadia, Phys. Rev. D 31, 3256 (1985).

[16] Ian Aitchison, Caroline Fraser, Elaine Tudor and Josef Zuk, Phys. Lett. B 165, 162 (1985).

[17] L. Marleau, J.F. Rivard, Phys. Rev. D 63, 036007 (2001)

[18] R.S.Ward, Nonlinearity 12, 241 (1999).

[19] Nicholas Manton and Paul Sutcliffe, Topological Solitons, (Cambridge University Press, 2004).

[20] M.Ostrogradski, Mem.Ac.St.Petersbourg 6, 185 (1850).

[21] A.Pais and G.E.Uhlenbeck, Phys. Rev. 79, 145 (1950).

[22] A.V.Smilga, Nucl. Phys. B 706, 598 (2005).

[23] D.A.Eliezer, R.P.Woodard, Nucl. Phys. B 325, 389 (1989).

[24] X.Jaén, L.Llosa and A. Morina, Phys. Rev.D 34, 2302 (1986).

[25] Jonathan Z.Simon, Phys. Rev. D 41, 3720 (1990); Phys. Rev. D 43, 3308 (1991). 